\title{
Impaired function of tendon-derived stem cells in experimental diabetes mellitus rat tendons: implications for cellular mechanism of diabetic tendon disorder
}

\author{
Liu SHI ${ }^{1,2,3,4,5+}$, Ying-juan LI ${ }^{4,5,6+}$, Guang-chun DAI 1,2,3,4,5 , Yu-Cheng LIN ${ }^{1,2,5}$, Gang LI , Chen WANG ${ }^{1,2,3,4,5,8}$,
} Hui CHEN ${ }^{1,2,3,4,5}$ and Yun-feng RUI 1,2,3,4,5,8,9*

\begin{abstract}
Background: Patients with diabetes mellitus (DM) often suffered with many musculoskeletal disorders, such as tendon rupture and tendinopathy. However, the understanding of the pathogenesis of these alternations is limited. This study was designed to investigate the role of tendon-derived stem cells (TDSCs) in histopathological alterations of DM tendons.

Methods: Forty-two SD rats were randomly and equally divided into a diabetes group (DG) and control group (CG). DM was induced by streptozotocin $(65 \mathrm{mg} / \mathrm{kg})$. The patellar tendons were isolated at weeks 1, 2, and 4 for histological analysis. TDSCs were isolated at week 2 for osteo-chondrogenic differentiation analysis. Mann-Whitney $U$ test was used with SPSS. $p<0.050$ was statistically significant.

Results: Micro-tears of collagen fibers and altered appearance of tendon cells were observed in DG tendons. DG tendons exhibited significantly higher expression of OPN, OCN, SOX9, and Col II and decreased expression of Col I and tenomodulin (TNMD) at week 2. Diabetic TDSCs (dTDSCs) demonstrated significantly decreased proliferation ability and increased osteogenic and chondrogenic differentiation ability. Osteo-chondrogenic markers BMP2, ALP, OPN, OCN, Col II, and SOX9 were also significantly increased while tenogenic markers Col I and TNMD were decreased in dTDSCs.

Conclusion: These results suggested the erroneous differentiation of dTDSCs might account for the structural and non-tenogenic alternations in DM tendons, which provided new cues for the pathogenesis of tendon disorders in DM.
\end{abstract}

Keywords: Diabetes mellitus, Tendinopathy pathogenesis, Tendon-derived stem cells, Erroneous differentiation

\section{Background}

With the increasing incidence of diabetes mellitus (DM) and the complications [1], such as retinopathy [2], nephropathy [3], and osteoporosis [4], numerous studies were performed to investigate the association between $\mathrm{DM}$ and these chronic diseases. However, little attention

\footnotetext{
* Correspondence: ruiyunfeng@126.com

${ }^{\dagger}$ Liu SHI and L. I. Ying-juan contributed equally to this work.

${ }^{1}$ Department of Orthopaedics, Zhongda Hospital, School of Medicine,

Southeast University, No. 87 Ding Jia Qiao, Nanjing 210009, Jiangsu, People's

Republic of China

${ }^{2}$ Orthopaedic Trauma Institute, Southeast University, No. 87 Ding Jia Qiao,

Nanjing 210009, Jiangsu, People's Republic of China

Full list of author information is available at the end of the article
}

was focused on the influences of DM on the tendons and ligaments. It is reported that the incidence of tendinopathy in patients with DM was significantly higher than healthy people at the same age $[5,6]$. Most of these patients suffered with the chronic pain and limited range of joint motion while some of the others were asymptomatic [7]. Increased thickness of diabetic tendons as well as the disorganized collagen fibers was found with ultrasound and magnetic resonance imaging [8, 9]. The deposition of the calcium at the enthesis of the Achilles tendons was also observed in several DM patients, thus resulted in higher risk of tendon rupture [10, 11]. In experimental DM animal models, the significantly

(c) The Author(s). 2019 Open Access This article is distributed under the terms of the Creative Commons Attribution 4.0 International License (http://creativecommons.org/licenses/by/4.0/), which permits unrestricted use, distribution, and 
decreased biomechanical properties of the DM tendons were also reported [12-15]. The weaken biomechanical properties might be attributed to the impaired healing process of the tendons in DM tendons with a smaller transverse area and less organized collagen fibers at the tendon-bone interface $[13,14]$. In gross view, the tendons in DM subjects showed a yellowish discoloration and much more fragile and atrophic [12,13]. The arrangement of the collagen fibers in DM tendons was in disorder when compared to the parallel and bundled healthy tendon fibers; the micro-tears were frequently observed as well [16].

In previous studies, we found tendon-derived stem cells (TDSCs) isolated from collagenase-induced tendinopathy rat model exhibited the increased osteo-chondrogenic differentiation potential and decreased tenogenic differentiation potential [17]. Therefore, taken together with the calcium deposition found in the Achilles tendons in DM patients [10], and such non-tenogenic changes in diabetic tendons have not been studied in depth, we raised the hypothesis that diabetic tendons have characteristics of structural and non-tenogenic changes as in classical tendinopathy, and the impaired function of TDSCs might account for these pathological changes, which might provide some implications for the cellular mechanism of the diabetic tendon disorders.

\section{Methods}

\section{Study design}

The 42 SD rats (female, 200-250 g, 8 weeks) were randomly divided into two groups: control group (CG, $n=$ 21), DM group (DG, $n=21$ ). At weeks 1,2 , and 4 post-induction, 6 rats of each group were sacrificed and the patellar tendons were isolated immediately for histological analysis. At week 2, 3 rats of both groups were sacrificed for TDSC isolation and analysis. All animal experiments were approved by the Animal Experimentation Ethics Committee, School of Medicine, Southeast University (Additional file 1).

\section{Induction of DM}

The method of rat DM model induction with streptozotocin (STZ; Sigma, St. Louis, MO) was well established $[12,18]$. Briefly, the rats were induced to DM through intraperitoneal injection of STZ-citrate buffer solution $(65 \mathrm{mg} / \mathrm{kg})$ while the CG rats received the citrate buffer only. All rats were fasted for $8 \mathrm{~h}$ and subjected to intraperitoneal glucose tolerance test (IPGTT) before and at day 3 post-injection. The sustained phenotype of DM is regarded as the maintained blood glucose (BG) levels equal to or higher than $250 \mathrm{mg} / \mathrm{dL}$ continuously. BG levels of all rats were measured every 2 days with a glucometer (ACCU-CHEK ${ }^{\circ}$ Performa).

\section{Histological analysis of the patellar tendons General histology and immunohistochemistry}

At weeks 1, 2, and 4 post-induction, the patellar tendons were isolated immediately after euthanasia and fixed in $4 \%$ paraformaldehyde for $24 \mathrm{~h}$. The tendons were dehydrated through gradient alcohol, embedded in paraffin, and cut longitudinally to $4-\mu \mathrm{m}$-thick sections. After deparaffination and hydration, the slides were stained with hematoxylin and eosin (H\&E). Immunohistochemistry (IHC) staining was performed as previously described [17, 19]; in brief, the primary antibodies against collagen type I (Col I, Abcam, Cambridge, USA; ab34710; 1:100), tenomodulin (TNMD, Santa Cruz Biotechnology, Santa Cruz, CA; sc-98875; 1:100), osteopontin (OPN, Abcam, Cambridge, USA; ab8448; 1:100), osteocalcin (OCN, Abcam, Cambridge, USA; ab13421; 1:100), SOX9 (Santa Cruz Biotechnology, Santa Cruz, CA; sc-20095; 1:30), and collagen type II (Col II, Abcam, Cambridge, USA; ab34712; 1:100) were used. Goat anti-rabbit (Abcam, Cambridge, USA; ab6721; 1:200) and goat anti-mouse IgG horseradish peroxidase (HRP)-conjugated secondary antibodies (Abcam, Cambridge, USA; ab6789; 1:200) were used according to the primary antibodies then chlorate with 3,3'-diaminobenzidine (DAKO, Glostrup, Denmark). Primary antibody was replaced with blocking solution in the controls. The slides were analyzed under a light microscope (Leica Cambridge, Cambridge, UK), semi-quantitative analysis of IHC staining was performed with Image-Pro Plus software (MediaCybernetics, Bethesda, MD, USA), and the assessors were blind to the sample grouping.

\section{Isolation and culture of TDSCs}

The procedures of TDSCs isolation and culture have been described previously [17]. Briefly, the middle substance of the patellar tendon was digested with type I collagenase $(3 \mathrm{mg} / \mathrm{ml}$, Sigma-Aldrich). The cells were cultured with the complete culture medium which contained low-glucose Dulbecco's modified Eagle's medium (Gibco), 10\% fetal bovine serum, $100 \mathrm{U} / \mathrm{mL}$ penicillin, $100 \mathrm{mg} / \mathrm{mL}$ streptomycin, and $2 \mathrm{mM} \mathrm{L}$-glutamine (all from Invitrogen Corporation, Carlsbad, CA). The early passages (P3 to P5) of both healthy TDSCs (hTDSCs) and diabetic TDSCs (dTDSCs) were used for all experiments.

\section{Flow cytometry assay}

For the flow cytometry assay, both hTDSCs and dTDSCs at P3 were used. $1 \times 10^{6}$ cells of each group were incubated with $1 \mathrm{mg}$ of phycoerythrin-conjugated or fluorescein-isothiocyanate-conjugated monoclonal antibodies (R\&D Systems) at $4{ }^{\circ} \mathrm{C}$ for $1 \mathrm{~h}$. Anti-CD34 (sc-7324; Santa Cruz Biotechnology, Santa Cruz, CA), anti-CD31 (ab33858; Abcam, Cambridge, UK), anti-CD44 (BD550974; BD Bioscience), anti-CD45 (BD559135; BD 
Bioscience), and anti-CD90 (BD554898; BD Bioscience) were the antibodies used in this study. Phycoerythrin-conjugated or fluorescein-isothiocyanate-conjugated isotypematched IgG1 were used as negative controls (IC002P or IC002F; R\&D Systems). The stained cells were washed with ice-cold PBS containing 2\% BSA before analysis using the LSRFortessa flow cytometer (Becton Dickinson, San Jose, CA). About $1 \times 10^{4}$ events were counted for each sample. The percentage of cells with a positive signal was calculated using the WinMDI Version 2.9 program (The Scripps Research Institute, La Jolla, CA).

\section{Colony forming ability assay and cell proliferation assay} For the colony-forming ability assay (CFA) [20], both hTDSCs and dTDSCs at P3 were seeded at the optimal cell density (1000 nucleated cells) on $20 \mathrm{~cm}^{2}$ dishes and cultured for 7 to 9 days to form colonies. The number of cell colonies was counted after stained with $0.5 \%$ crystal violet (Sigma, St Louis, MO). total number of colonies of both groups were recorded after ignoring the colonies which were less than $2 \mathrm{~mm}$ in diameter and were faintly stained.

For the cell proliferation assay, the P3 cells of both groups were seeded on a 96-well plate (4000 cells/well). After incubated for 24, 48, 72, and $96 \mathrm{~h}$, cells were treated with methyl thiazolyl tetrazolium (MTT) solution $(0.5 \mathrm{mg} / \mathrm{ml})$ for $4 \mathrm{~h}$ then dissolved with dimethyl sulfoxide and shaken for $10 \mathrm{~min}$. The absorbance was measured at $570 \mathrm{~nm}$ with a microplate reader.

\section{Osteo-chondrogenic differentiation potential of hTDSCs and dTDSCs \\ Osteogenic differentiation assay}

The methods of TDSC multi-differentiation induction have been described in the previous studies [17, 20]. For the osteogenesis differentiation assay, two kinds of TDSCs were plated at $4 \times 10^{3}$ cells $/ \mathrm{cm}^{2}$ in 6 -well plate and cultured in a complete culture medium until the cells reached 80 to $90 \%$ confluence then incubated in both basal medium and osteogenic induction medium (OIM) which was complete culture medium supplemented with $20 \mathrm{mM} \beta$-glycerolphosphate, $50 \mathrm{mM}$ ascorbic acid, and $1 \mathrm{nM}$ dexamethasone (all from SigmaAldrich) for 7 days. Alkaline phosphatase (ALP) staining and the mRNA expression of ALP, bone morphogenetic protein 2 (BMP2), OPN, and $\mathrm{OCN}$ by quantitative real-time reverse transcription-polymerase chain reaction (qRT-PCR) assay were performed. Alizarin red staining (ARS) was done after the basal medium and OIM incubated for 14 days to evaluate the calcium nodule formation.

\section{Chondrogenic differentiation assay}

The pellet culture system was used for chondrogenic differentiation assay. $8 \times 10^{5}$ cells (P3) were used to form a pellet in a $15-\mathrm{mL}$ conical polypropylene tube by centrifugation at $450 \mathrm{~g}$ for $10 \mathrm{~min}$ and cultured in both basal medium and chondrogenic induction medium (CIM) which contained low-glucose Dulbecco's modified Eagle's medium (Gibco, Invitrogen Corporation) supplemented with $500 \mathrm{ng} / \mathrm{mL}$ BMP-2 (R\&D Systems, Inc.), $10 \mathrm{ng} / \mathrm{mL}$ transforming growth factor- $\beta 3$ (R\&D), $50 \mathrm{mg} / \mathrm{mL}$ ascorbate-2-phosphate, $10^{-7} \mathrm{M}$ dexamethasone, $40 \mathrm{mg} /$ $\mathrm{mL}$ proline, $100 \mathrm{mg} / \mathrm{mL}$ pyruvate (all from Sigma-Aldrich), and 1:100 diluted ITS + Premix (6.25 $\mathrm{mg} / \mathrm{mL}$ insulin, $6.25 \mathrm{mg} / \mathrm{mL}$ selenous acid, $6.25 \mathrm{mg} / \mathrm{mL}$ transferrin, $5.35 \mathrm{mg} / \mathrm{mL}$ linoleic acid, $1.25 \mathrm{mg} / \mathrm{mL}$ bovine serum albumin) (Becton Dickinson, Franklin Lakes, $\mathrm{NJ})$ at $37^{\circ} \mathrm{C}, 5 \% \mathrm{CO}_{2}$. At day 28 , the pellets were either fixed for histological analysis (H\&E and Safranin O (SO)) and IHC staining (SOX9 and Col II) or total RNA extraction for mRNA expression as described below.

\section{mRNA expression of tenogenic markers}

Both hTDSCs and dTDSCs at P3 were seeded at $1 \times 10^{4}$ cells $/ \mathrm{cm}^{2}$ in a 6-well plate in the complete culture medium at $37^{\circ} \mathrm{C}, 5 \% \mathrm{CO}_{2}$. At day 7 , the cells were harvested and the mRNA expression of tenogenic markers, including Col I, scleraxis (Scx), and TNMD were examined by qRT-PCR as described below.

\section{qRT-PCR assay}

qRT-PCR was performed as previously described [21]. Briefly, cells were harvested and homogenized for RNA extraction with RNeasy mini kit (Qiagen $\mathrm{GmbH}$, Hilden, Germany). Then, mRNA was reverse transcribed to cDNA by the First-Strand cDNA kit (Promega, Madison, WI). The condition for PCR was optimized in a conventional PCR machine (GeneAmp 9700; Applied Biosystems, Foster City, CA) for the primers specific for GAPDH, BMP2, OPN, OCN, Col II, and SOX9 as shown in Table 1 at various annealing temperatures (Table 1). Cycling conditions were as follows: denaturation at $95^{\circ} \mathrm{C}$ for $10 \mathrm{~min}, 45$ cycles at $95^{\circ} \mathrm{C}$ for $20 \mathrm{~s}$, optimal annealing temperature for $30 \mathrm{~s}, 72^{\circ} \mathrm{C}$ for $30 \mathrm{~s}$, and finally at $60-95^{\circ} \mathrm{C}$ with a heating rate of $0.1{ }^{\circ} \mathrm{C} / \mathrm{s}$. Results were analyzed using ABI StepOne Plus system (Applied Biosystems, Foster City, CA). Expression of the target gene was normalized to GAPDH gene. Relative gene expression was calculated using the $2^{-\Delta \Delta \mathrm{CT}}$ formula.

\section{Statistical analysis}

The data was presented in histogram and reported as mean \pm standard deviation. Mann-Whitney $U$ test was done using SPSS (SPSS, Inc., Chicago, IL; version 16.0). $\quad p<0.050$ was considered as statistically significant. 
Table 1 Primer sequences and condition for qRT-PCR

\begin{tabular}{|c|c|c|c|c|}
\hline Gene & Primer nucleotide sequence & Product size (bp) & Annealing temperature & Accession no \\
\hline GAPDH & $\begin{array}{l}\text { 5'-CGGCAAGTTCAACGGCACAG-3' (forward) } \\
\text { 5'-GAAGACGCCAGTAGACTCCACGAC-3' (reverse) }\end{array}$ & 148 & 60 & NM_017008.4 \\
\hline BMP2 & $\begin{array}{l}\text { 5'-CCCTITGTATGTGGACTTCAGTGATGTG-3' (forward) } \\
\text { 5'-CTATGGCATGGTTGGTGGAGTTCAG-3' (reverse) }\end{array}$ & 137 & 63 & NM_017178.1 \\
\hline OPN & $\begin{array}{l}\text { 5'-CAGTCGATGTCCCTGACGG-3' (forward) } \\
\text { 5'-GTTGCTGTCCTGATCAGAGG-3' (reverse) }\end{array}$ & 206 & 60 & NM_012881.2 \\
\hline OCN & $\begin{array}{l}\text { 5'-GGTGCAAAGCCCAGCGACTCT-3' (forward) } \\
\text { 5'-GGAAGCCAATGTGGTCCGCTA-3' (reverse) }\end{array}$ & 199 & 60 & NM_013414.1 \\
\hline ALP & $\begin{array}{l}\text { 5'-ACCATTCCCACGTCTTCACATTT-3' (forward) } \\
\text { 5'-AGACATTCTCTCGTTCACCGCC-3' (reverse) }\end{array}$ & 162 & 60 & NM_013059.1 \\
\hline Coll & $\begin{array}{l}\text { 5'-CATCGGTGGTACTAAC-3' (forward) } \\
\text { 5'-CTGGATCATATTGCACA-3' (reverse) }\end{array}$ & 238 & 55 & NM_053356.1 \\
\hline TNMD & $\begin{array}{l}\text { 5'-CCATGCTGGATGAGAGAGGTTAC-3' (forward) } \\
\text { 5'-CACAGACCCTGCGGCAGTA-3' (reverse) }\end{array}$ & 72 & 58 & NM_022290.1 \\
\hline Scx & $\begin{array}{l}\text { 5'-AACACGGCCTTCACTGCGCTG-3' (forward) } \\
\text { 5'-CAGTAGCACGTTGCCCAGGTG-3' (reverse) }\end{array}$ & 102 & 58 & NM_001130508.1 \\
\hline Col II & $\begin{array}{l}\text { 5'-GAGTGGAAGAGCGGAGACTACTG-3' (forward) } \\
\text { 5'-CTCCATGTTGCAGAAGACTTTCA-3' (reverse) }\end{array}$ & 81 & 55 & NM_012929.1 \\
\hline Sox9 & $\begin{array}{l}\text { 5'-CTGAAGGGCTAGGACTGGAC-3' (forward) } \\
\text { 5'-TACTGGTCTGCCAGCTCCT-3' (reverse) }\end{array}$ & 140 & 58 & NM_080403.1 \\
\hline
\end{tabular}

\section{Results}

\section{Induction of DM}

The rats treated with STZ solution demonstrated that a sustained phenotype of DM with mean BG value in DG subjects was sustained higher than $250 \mathrm{mg} / \mathrm{dL}$ (Table 2). The mean area under the curve (AUC) in IPGTT of DG subjects also significantly increased when compared with the controls 3 days post-STZ injection $(22,530 \pm 2190$ and $8956 \pm 1126$, respectively) $(* * p=0.002)$, which provided a quantitative index of the severity of hyperglycemia in DG subjects (Table 2), thus indicating the sustained hyperglycemia of DG rats after STZ injected.

\section{Macroscopy observation of pathological changes Histopathology of the patellar tendons in DG rats}

The patellar tendons in DG subjects exhibited disorder arrangement of collagen fibers when the controls demonstrated the tight, parallel arrangement with slight waves (Fig. $1(1 \mathrm{~A}-1 \mathrm{~F}))$. What is more, the micro-tears were frequently observed in diabetic tendons at weeks 2 and 4 post-induction (Fig. 1 (1E, 1E), arrow). The red blood cells and small blood vessels (Fig. 1 (1E, 1E), rectangle) and the rounding changed tendon cells surrounding the tear sites were observed in the diabetic tendons at weeks 2 and 4 post-induction (Fig. 1 (1E, 1E),

Table 2 Results of BG value and IPGTT of both CG and DG rats

\begin{tabular}{lll}
\hline & BG level $(\mathrm{mg} / \mathrm{dL})$ & Mean AUC \\
\hline CG & $76.3 \pm 1.5$ & $8643.6 \pm 395.2$ \\
DG & $344.7 \pm 13.9^{*}$ & $22,361.2 \pm 1009.8^{*}$ \\
\hline
\end{tabular}

${ }^{*} p<0.01$ compared with CG diamond) while healthy tendon cells in slender shape were well distributed within the longitudinally arranged collagen fibers. These results suggest the DG patellar tendons exhibited the pathological alternations at the early stage of STZ-induced DM rats.

\section{IHC staining of the patellar tendons}

Expression of osteo-chondrogenic differentiation markers

The expression of osteogenic differentiation markers, including OPN and OCN, were positively stained in the DG tendons while the CG group showed to be negative (Fig. $2(2 \mathrm{~A}-2 \mathrm{H}$, $2 \mathrm{a}-2 \mathrm{~h}))$. OPN and OCN were mainly expressed in the cytoplasm of diabetic tendon cells, especially surrounding these rounded tendon cells (Fig. 2 (2a-2f), arrow). The chondrogenic differentiation markers, including SOX9 and Col II, were also positively expressed in the diabetic tendon while the CG group showed the negatively stained (Fig. 2 (2G-2L, $2 \mathrm{~g}-2 \mathrm{l})$ ). SOX9 was found positively stained in both the nucleus and the cytoplasm of these cells while Col II mainly expressed in the extracellular matrix of tendon cells (Fig. 2 (2g-2l), arrow). These significantly increased osteo-chondrogenic differentiation markers in DG tendon further illustrated the pathological changes in STZ-induced DM rats.

\section{Expression of tenogenic markers}

All the patellar tendons of both DC and CG subjects showed positive expression of Col I and TNMD (Fig. 3 $(3 \mathrm{~A}-3 \mathrm{~F}, 3 \mathrm{a}-3 \mathrm{f}))$. The semi-quantitative analysis of IHC staining showed the significantly decreased mean integrated optical density (IOD) $/ \mu^{2}$ of Col I and TNMD in the patellar tendon of the DG at week 2 post-induction when 


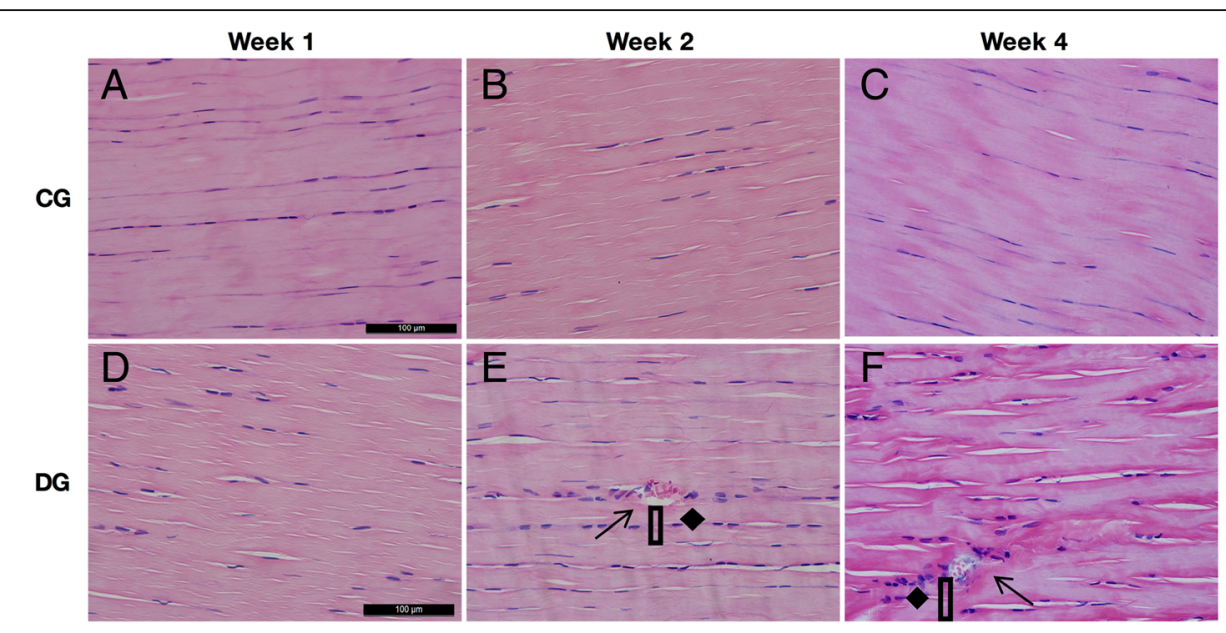

Fig. 1 Representative images of H\&E staining of both CG and DG patellar tendons at weeks 1, 2, and 4 post-STZ induction. 1A-1C: All of the CG tendons at different time points showed the tight, parallel arrangement of collagen fibers distributed at the same orientation with slight waves. The bundles of collagen fiber were about the same size as well as the tendon cells. 1D-1F: Alternations of collagen fibers arrangement, microtears of collagen fibers (arrow), the rounded tendon cells (diamond), and even some red blood cells and blood vessels (rectangle) were observed in diabetic tendons. $n=6$ of each group. Scale bar $=100 \mu \mathrm{m}$

compared with the controls (Fig. $3(3 \mathrm{M}, 3 \mathrm{~N}),{ }^{*} p=0.010$ and $* * 0.004$, respectively). Interestingly, the expression of Col I in diabetic tendons was remarkably increased at week 4 than that of week 2 (Fig. $3(3 \mathrm{M})$, " $p=0.010$ ). These histological and IHC results demonstrated the tendons in STZ-induced DG rats exhibited the characteristics of structural and non-tenogenic alternations as in classical tendinopathy.

\section{Immunophenotypes and proliferative capacity of TDSCs}

TDSCs were isolated from both CG and DG rat patellar tendons after 2 weeks post-STZ induction in this study.

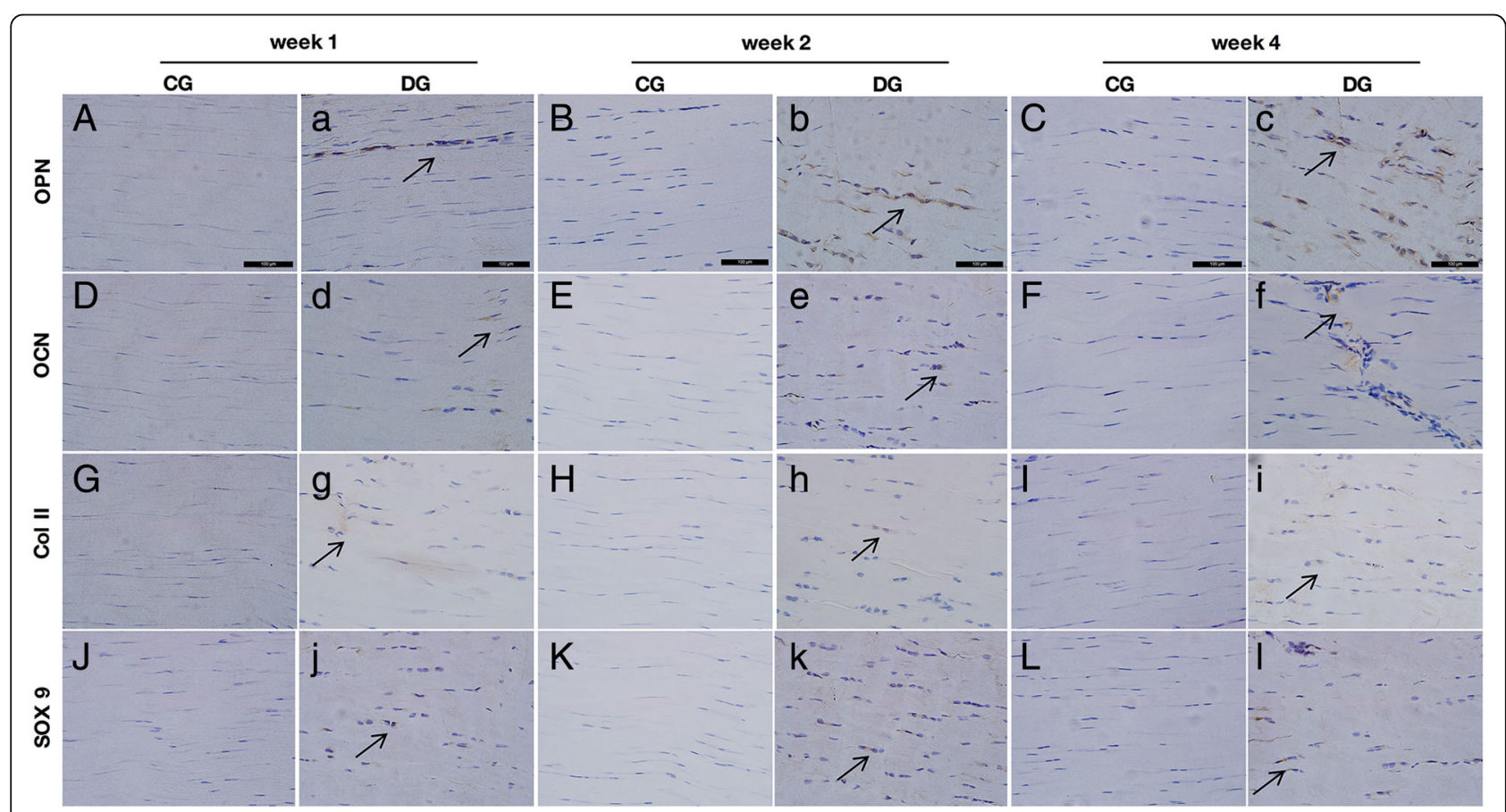

Fig. 2 Representative images of IHC staining of osteo-chondrogenesis markers, OPN, OCN, Col II, and SOX9 in CG and DG tendons at weeks 1, 2, and 4 post-STZ induction. The osteogenic markers OPN and OCN were both positively expressed in DG tendons (2a-2f, arrows) at weeks 1, 2, and 4 while the controls were negatively expressed (2A-2F). The chondrogenesis markers Col II and SOX9 were also positively expressed in DG tendons ( $2 \mathrm{~g}-2 \mathrm{l}$, arrows) at weeks 1,2 , and 4 while the controls were negatively expressed (2G-2L). $n=6$ of each group. Scale bar $=100 \mu \mathrm{m}$ 

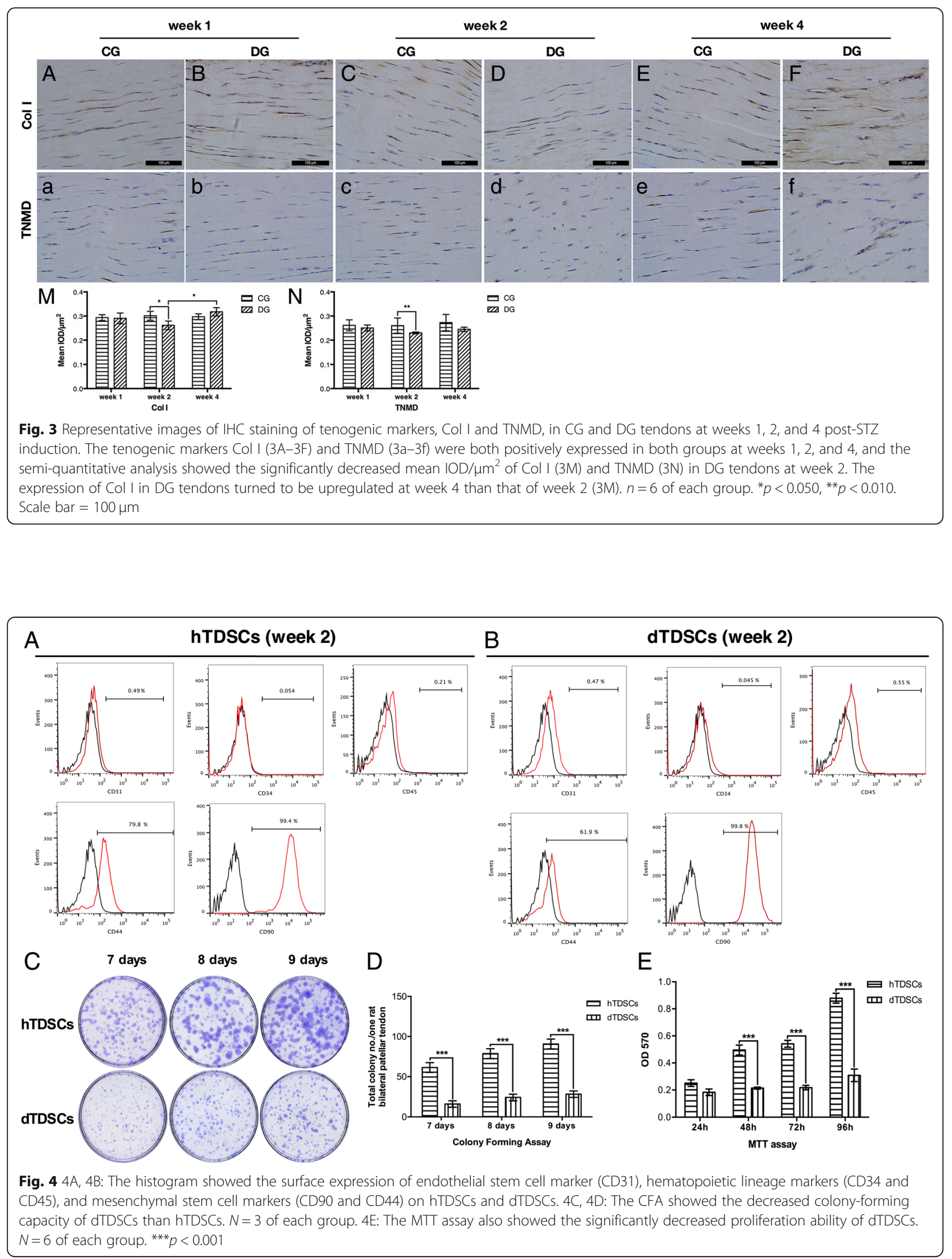
Both hTDSCs and dTDSCs were positive for mesenchymal stem cell markers, including CD90 and CD44, but negative for hematopoietic lineage markers, including CD34 and CD45, and endothelial cell markers, including CD31. The surface expression of CD44 was remarkably reduced in dTDSCs (61.9\%) compared to hTDSCs (79.8\%) (Fig. 4 (4A, 4B). Less TDSC colonies were found in DG group at 7,8 , and 9 days when compared with matched controls (Fig. 4 (4C, 4D), all **** $p<0.001$ ). In addition, the results of MTT reduction assay also suggested the significantly decreased proliferative ability of dTDSCs at 48, 72, and 96 h (Fig. 4 (4E), all ${ }^{* * *} p<0.001$ ).

\section{Osteogenic differentiation potential}

In the osteogenic differentiation assay, the dTDSCs showed significantly higher ALP activity than the hTDSCs at day 7 under both basal medium and OIM incubation (Fig. 5 (5A), $* * p=0.002$ and $* *=0.002$, respectively). Meanwhile, more ARS-positive calcium nodules were found in dTDSCs at day 14 post-basal medium and OIM incubation. The quantification analysis also showed the dTDSCs exhibited a significantly higher intensity of the calcium-bound ARS signal at day 14 in both basal and OIM when compared (Fig. 5 (5A), $* p=0.004$ and $* * p=0.001$, respectively). What is more, the expression of ALP $(* * * * 0<0.001)$, BMP2 $(* * p<0.001)$, OPN (" $p=0.030)$, and OCN (" $p=0.020)$ of dTDSCs also remarkably upregulated at mRNA level when compared with the hTDSCs under OIM incubation for 7 days (Fig. 5 (5D)). The mRNA expression of ALP $(* * * * 0<0.001)$, BMP2 (*** $p=0.004)$, and OPN (* $p=0.022)$ of dTDSCs were significantly increased as well under basal medium incubation for 7 days (Fig. 5 (5C)). Taken together, these findings indicated that the osteogenic differentiation potential of TDSCs in STZ-induced DM rats was upregulated in vitro.

\section{Chondrogenic differentiation potential}

In the chondrogenic differentiation assay, both hTDSCs and dTDSCs formed pellets at day 28 after chondrogenic induction in a 3D pellet culture system while these induced in basal medium failed to form pellets. The pellet of dTDSCs was much larger than that of hTDSCs in gross view (Fig. 6 (6A)). More chondrocyte-like cells (Fig. 6 (6B, $6 \mathrm{C})$ ) and much higher expression of proteoglycan deposition were detected (Fig. 6 (6D, 6E)) in the dTDSCs group than the hTDSCs group. What is more, the expression of Col II and SOX9 was also significantly increased in dTDSCs with IHC staining (Fig. 6 (6F-6I, 6L), " $p=0.050$ and ${ }^{*} p=0.050$, respectively). The qRT-PCR data also demonstrated that the expression of both Col II and SOX9 in dTDSCs was significantly upregulated at mRNA level under CIM induction for 7 days (Fig. $6(6 \mathrm{~N}), " p=0.016$ and $" p=0.030$, respectively), and the expression of Col II was also increased in dTDSCs under basal medium incubation (Fig. $6(6 \mathrm{~N}), " p=0.011)$. All these findings

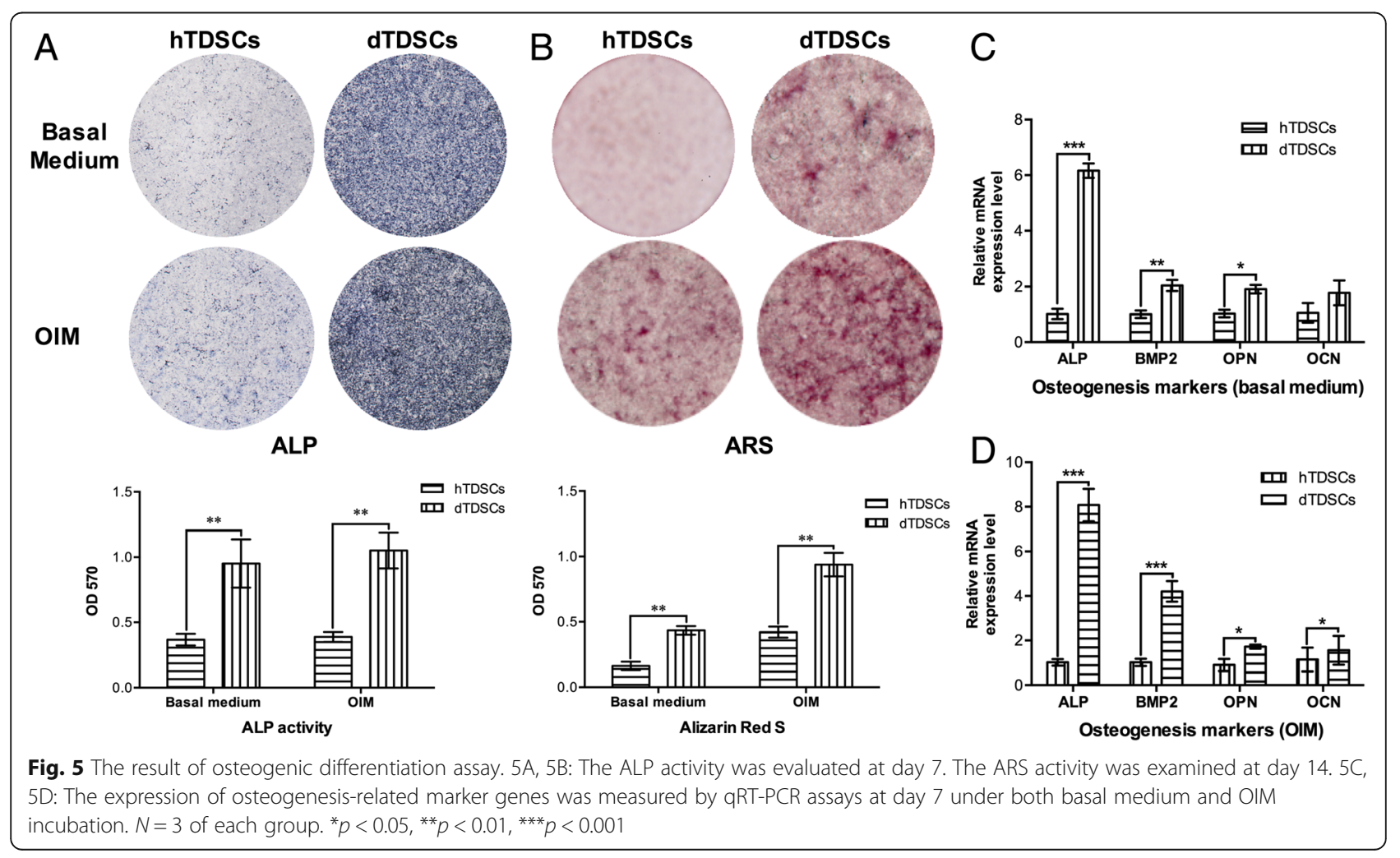




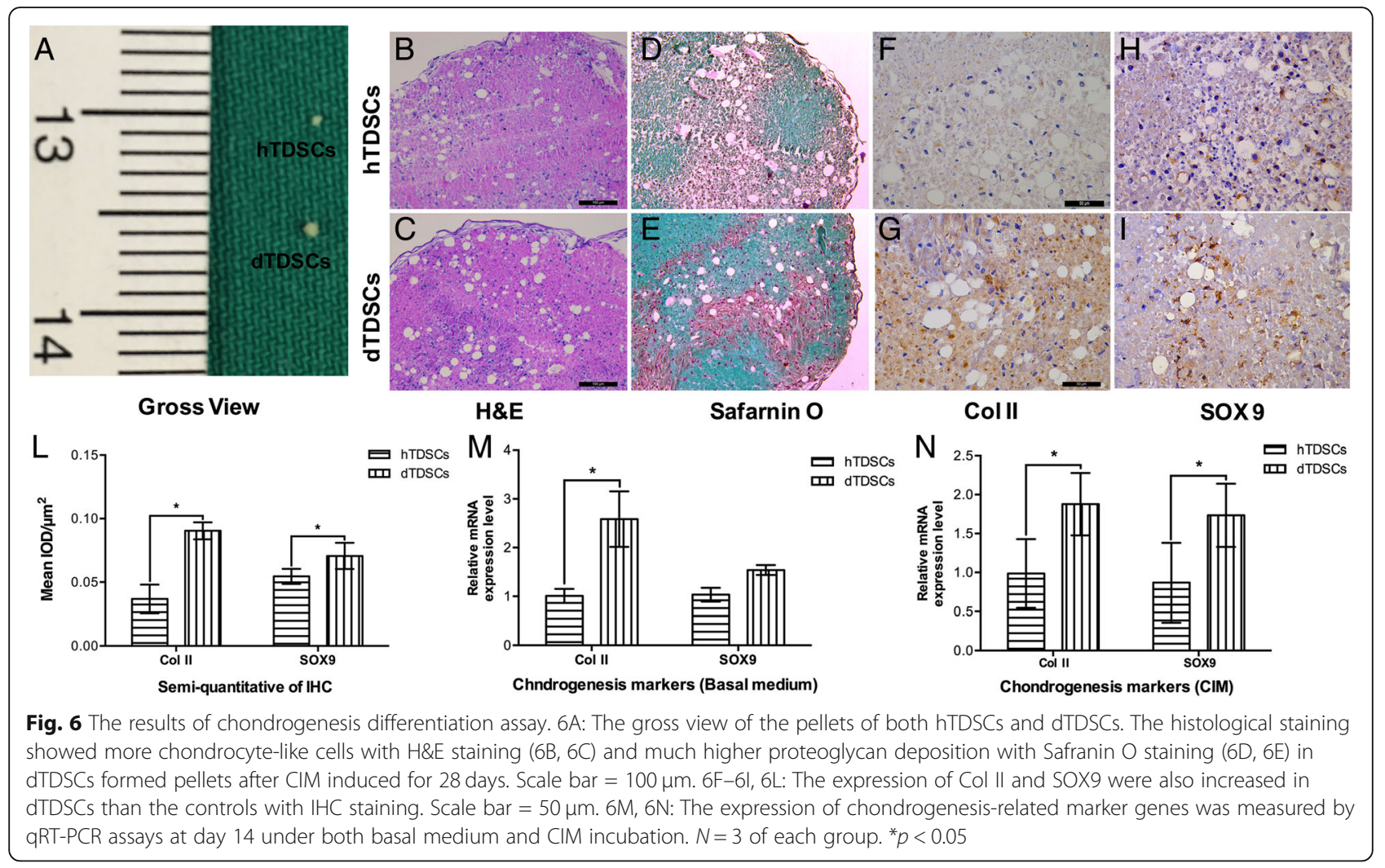

suggested the dTDSCs possessed higher chondrogenic differentiation potential than hTDSCs.

\section{Expression of tenogenic markers}

The tenogenic ability of hTDSCs and dTDSCs was compared by investigating the mRNA expression of tenogenic markers, including Col I, TNMD, and scleraxis (Scx). There was a significantly lower mRNA expression of both Col I (Fig. 7 (7A), ${ }^{*} p=0.041$ ) and TNMD (Fig. 7 $\left.(6 \mathrm{~B}),{ }^{* * *} p=0.002\right)$ in dTDSCs when compared with the hTDSCs in basal medium. Interestingly, the expression of Scx was observed to be significantly increased in
dTDSCs when compared with the hTDSCs in basal medium (Fig. 7 (6C), "*" $p=0.002$ ).

\section{Discussion}

The underlying mechanism of the pathological changes in diabetic tendons still remained unclear $[6,22]$. In the present study, we investigated the non-tenogenic histopathological alternations in the patellar tendon of experimental type I DM rats and the properties of the TDSCs in the diabetic tendon.

STZ could enter the pancreatic islet B cells via a glucose transporter and cause rapid destruction of B cells and induced subjects to type I DM [18]. Therefore, it is
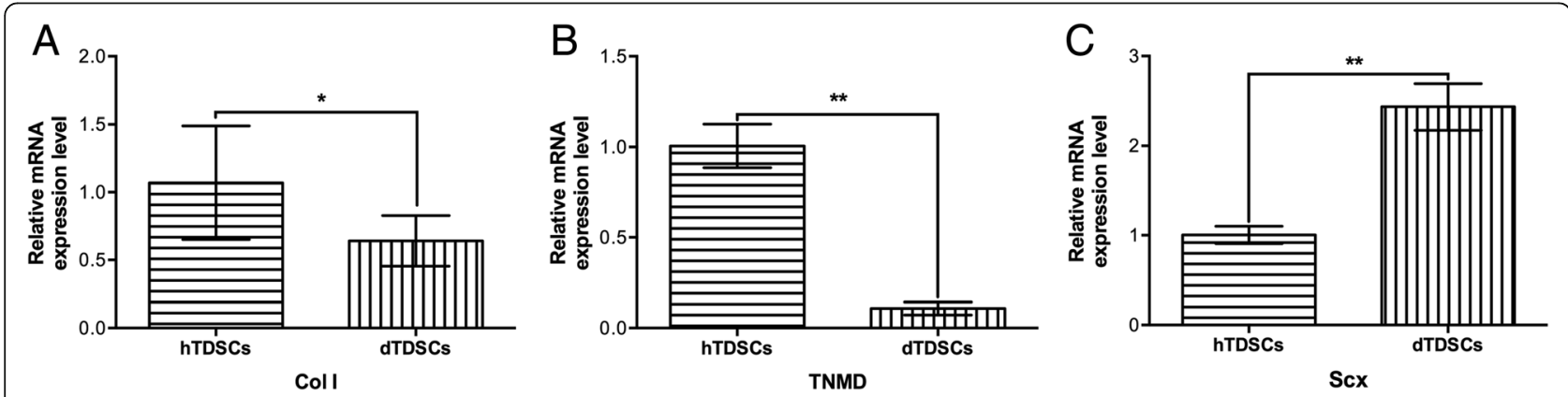

Fig. 7 The qRT-PCR results of tenogenic markers. 7A, 7B: The significantly decreased Col I and TNMD expression in dTDSCs than the controls. 7C: The expression of Scx was significantly upregulated in dTDSCs than the hTDSCs. $N=3$ of each group. ${ }^{*} p<0.050,{ }^{* *} p<0.010$ 
widely used to establish the DM animal model in many studies [12, 18]. After injected STZ solution for 3 days, the results of IPGTT indicated the diabetes phenotype of DG rats in our study and the sustained higher BG level also showed the DM model was successfully established.

In the current study, the histological analysis showed the characteristic tendinopathy alternations in diabetic tendons including the unparalleled arrangement of collagen fibers, disorganized tendon cells in the extracellular matrix, and the rounding changed tendon cells (Additional file 2). The micro-tears, red blood cells, and blood vessels were also discovered in DG tendons while the vessels could not be found in CG tendons. The increased number of vessels in tendons of both DM patients and animal models was also reported [14]. They discovered that the cross-sectional area of these proliferous vessels was also larger, and the vascular endothelial growth factor (VEGF) was also significantly increased in DM tendons [14]. Scientific evidences showed the good blood supply and innervation were essential for the collagen synthesis formation at the injured sites of tendons [23]. Therefore, the increased angiogenesis and upregulated VEGF expression also indicated the formation of chronic tendinopathy in DM subjects. In addition, the deposition of calcium in the Achilles tendon of patients with DM was reported in the clinical study [10]. The fibrocartilage metaplasia was also significantly increased in DM tendons accomplished with stenosing flexor tenosynovitis when compared with non-diabetic subjects [24]. Local hypoxia which might be induced by the micro-tears of collagen fibers and disturbance of micro-circulation were the factors that might contribute to the calcification in DM tendons [25]. These alternations implied the degeneration of DM tendons. These degenerative features might lead to the decreased biomechanical properties of DM tendons when compared to healthy subjects and further resulted in the chronic pain and tendon rupture in some DM patients [11-13].

Furthermore, the positive expression of osteo-chondrogenic markers, including OPN, OCN, Col II, and SOX9, was observed in diabetic tendons while the CG tendons showed the negative expression, thus indicating some of the tendon cells in DM tendons exhibited higher non-tenogenic differentiation potential in vivo. Moreover, the expression tenogenic markers (Col I and TNMD) was decreased in diabetic tendons at week 2 when compared to matched controls. In our previous study, we found the proliferation ability of TDSCs was significantly decreased after treated with high glucose $(15 \mathrm{mM}(270 \mathrm{mg} / \mathrm{dl})$ and $25 \mathrm{mM}$ $(450 \mathrm{mg} / \mathrm{dl}))$ for 1 and 3 days, and the tendon-related markers were also downregulated in vitro [26]. In DM tendon healing models, the proliferation potential of the DM rat tendon cells was also declined in vitro, and the synthesis of Col I and type III collagen of DM tendon was decreased after injury as well [15]. Taken together, these results suggested that the patellar tendon in STZ-induced DM rats might suffer a transitorily decrease of the collagen production at the early stage of the experimental type I DM subjects.

TDSCs exhibit universal stem cell characteristics, including clonogenicity, proliferative capacity, multi-differentiation potential, and mesenchymal stem cell marker expression [20]. Erroneous differentiation of TDSCs towards osteogenesis and chondrogenesis was reported to be a pathogeny for chronic tendinopathy [17]. Hence, we speculate the fate of TDSCs in DM rats was also changed and the erroneous differentiation of these stem cells might lead to the chondrocyte-like change of the tendon cells and the calcium deposition in these tendons.

According to the histological and IHC results, the patellar tendons showed the declining collagen production than the controls after STZ injection for 2 weeks; thus, we isolated the TDSCs at this time point in order to dig out the possible changes at cellular level. Both hTDSCs and dTDSCs positively express mesenchymal stem markers including CD90 and CD44, and the expression of CD44 in dTDSCs was decreased than hTDSCs, as a cell-surface glycoprotein, CD44 participates in cell growth, survival, differentiation, and motility processes [27]. It is reported that knockdown of CD44 could lead to the promoted differentiation of cancer stem cells [28]. Thus, the reduction of CD44 expression in dTDSCs might be related to the altered multi-differentiation potential.

Our results showed the significantly decreased proliferation capacity of dTDSCs when compared to the hTDSCs in vitro; this was consistent with the previous finding that high glucose could suppress the proliferation ability of TDSCs in vitro [26]. What is more, the upregulated ALP activity, calcium deposition, and higher proteoglycan deposition in dTDSCs formed pellets and increased mRNA expression of osteogenesis and chondrogenesis markers, suggesting the significantly enhanced osteo-chondrogenesis differentiation potential of dTDSCs than that of hTDSCs. In addition, we also observed the downregulated tenogenic markers Col I and TNMD expression at mRNA level. Previously, we also found the TDSCs treated with high glucose for 24 and $28 \mathrm{~h}$ showed significantly decreased TNMD and Col I expression in vitro [26]. The spontaneous tenogenic differentiation was also reported to be a specific feature of TDSCs [29], manifested the important role of TDSCs in tendon metabolism and repair. However, the dTDSCs showed the decreased tenogenic differentiation potential with significantly increased osteo-chondrogenic differentiation potential than the hTDSCs at week 2 post-STZ induction; this might account for the decreased collagen 
production and increased non-tenogenic alternations in DM tendons.

Interestingly, the expression of Scx in dTDSCs was significantly upregulated simultaneously. As a transcriptional regulator, Scx is abundantly expressed in embryonic and adult tendons and reported to be re-expressed after tendon injury [30]. The increased expression of Scx might be due to the chronic tendinopathy in DM tendons; the micro-tears of tendon fibers in DM tendons and decreased collagen expression in the extracellular matrix might lead to the upregulated expression of Scx in transcription level [31]. Taken together, the upregulated osteo-chondrogenesis and downregulated tenogenic differentiation potential of TDSCs in DM tendons might be contributed to the increased expression of OPN, OCN, Col II, and SOX9 and decreased expression of Col I and TNMD in diabetes tendons with IHC staining and further resulted in the decreased mechanical properties and degenerative changes, even characteristic tendinopathy alternations in DM tendons.

Nevertheless, the limitations in this study were that the stem cells which we isolated from both control and DM rats could be exogenous or endogenous since the specific marker for TDSCs was not well established, and the composition of the TDSCs was also up to the pathological status of the rats. The other was the direct influence of STZ on tendons, and TDSCs are not studied in depth. It is reported the chronic effect of STZ on tendon mechanical properties in DM animals was due to the high glucose level in vivo after STZ injection [12, 32]. Lehner et al. reported that tendons in non-diabetic human and rats contained a population of pancreas beta cell-like cells which also producing insulin and glucose transporter 2 (GLUT2) [33]. GLUT2 is a key protein which could also transport STZ into the cells [34]; thus, they thought STZ could reduce tendon mechanical strength by affecting tendon cells after a single IP injection of STZ for 5 days. However, they treated the tendons with STZ and showed no apparent structural alterations in vitro. What is more, we also treated the TDSCs with STZ; the osteogenic differentiation potential of TDSCs was not affected in vitro during OIM induction. The expression of GLUT2 was not increased after STZ is added. Therefore, we speculate the primary reason for the activation of these GLUT2-expressing cells in tendon tissue might be due to the systematic increased high glucose level in vivo.

In our previous study, we investigated the direct effects of high glucose on TDSCs in vitro and discovered the expression of tenogenic markers including Tnmd and Col I in TDSCs treated with high glucose were significantly decreased. Actually, we have not investigated the effects of high glucose on the osteo-chondrogenic differentiation of TDSCs.

It is reported that various signaling pathways, such as WNT/ $\beta$-catenin and TGF- $\beta$, were activated in the osteogenic differentiation of tendon-derived cells and TDSCs $[35,36]$. The $\mathrm{WNT} / \beta$-catenin signaling pathway in MSCs osteogenic differentiation was well studied [37]. Therefore, the enhanced osteogenic differentiation in dTDSCs might be attributed to the triggering of WNT/ $\beta$-catenin; the molecular mechanism of these observed

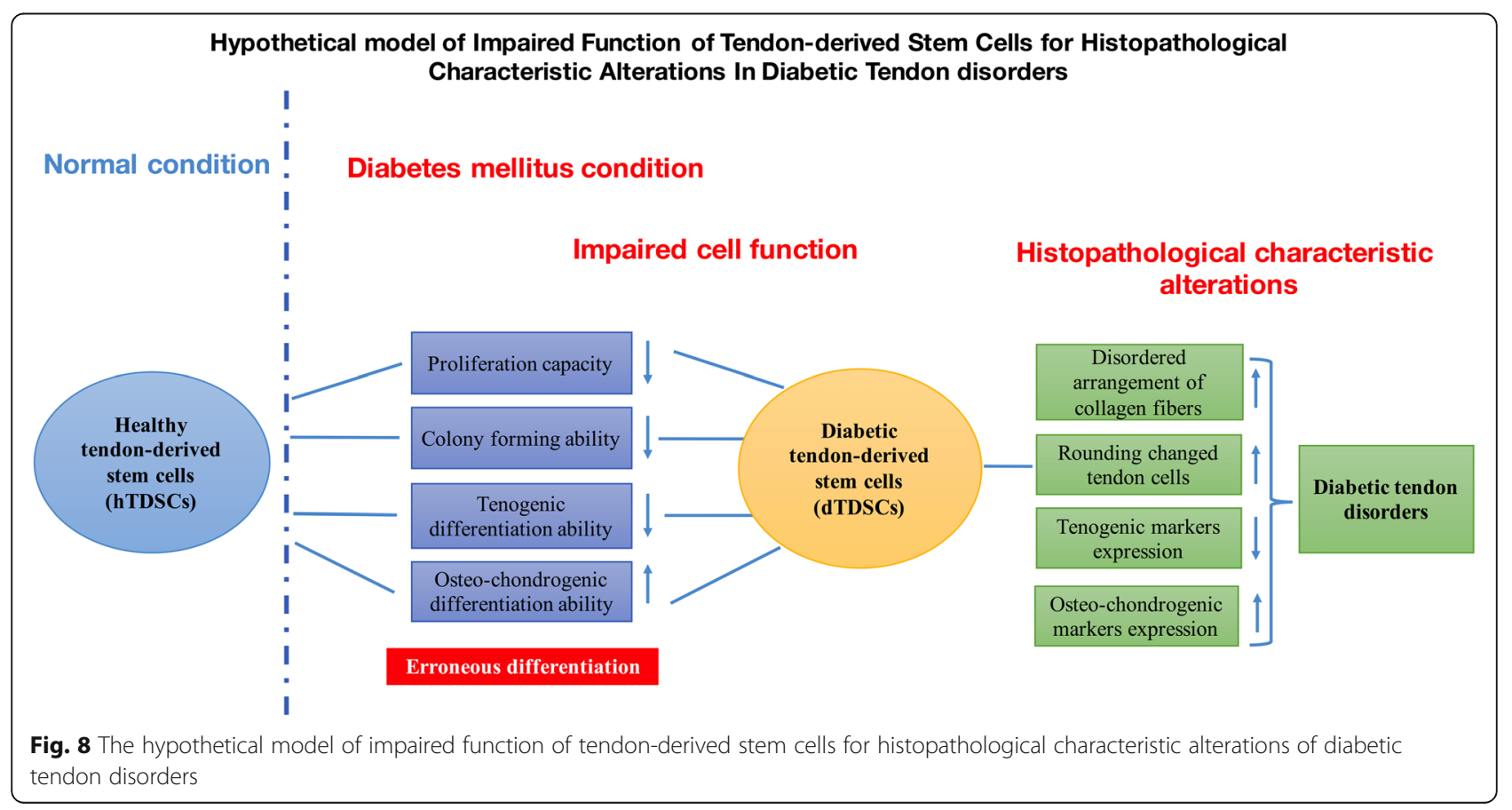


erroneous differentiation of TDSCs and dTDSCs in our previous and present studies needs further investigation.

\section{Conclusions}

In summary, we could also conclude that the dTDSCs which isolated from STZ-induced DM rats showed the significantly decreased proliferation capacity and colony-forming ability than the normal healthy TDSCs, and the erroneous differentiation of dTDSCs with higher osteo-chondrogenic and lower tenogenic differentiation abilities might contribute to the non-tenogenic alternations of tendons in STZ-induced DM rats, further impair its self-managing ability, and develop into histopathological characteristic alterations of diabetic tendon disorders (Fig. 8). Further studies are also needed to explore the potential molecular mechanism of the pathological alternations in DM tendons.

\section{Additional files}

Additional file 1: Ethics approval for animal experimentation. This is the file of the ethics approval for animal experimentation in School of Medicine, Southeast University. (PDF 362 kb)

Additional file 2: Polarizing microscopy view. The typical collagen birefringence was lost in DG tendons when compared with the CG tendons at week 4 under polarizing microscope. (PNG 266 kb)

\section{Abbreviations}

ALP: Alkaline phosphatase; ARS: Alizarin red staining; AUC: Area under the curve; BG: Blood glucose; BMP2: Bone morphogenetic protein 2; CFA: Colony-forming ability assay; CIM: Chondrogenic induction medium; Col I: Collagen type I; Col II: Collagen type II; DM: Diabetes mellitus; dTDSCs: Diabetic TDSCs; GLUT2: Glucose transporter 2; H\&E: Hematoxylin and eosin; HRP: Horseradish peroxidase; hTDSCs: Healthy TDSCs; IHC: Immunohistochemistry; IOD: Integrated optical density; IPGTT: Intraperitoneal glucose tolerance test; MTT: Methyl thiazolyl tetrazolium; OCN: Osteocalcin; OIM: Osteogenic induction medium; OPN: Osteopontin; qRT-PCR: Quantitative real-time reverse transcriptionpolymerase chain reaction; Scx: Scleraxis; SO: Safranin O; STZ: Streptozotocin; TDSCs: Tendon-derived stem cells; TNMD: Tenomodulin; VEGF: Vascular endothelial growth factor

\section{Funding}

This work was supported by the National Natural Science Foundation of China for Young Scholars (81201422); National Natural Science Foundation of China (81572187, 81871812); China Postdoctoral Science Foundation (2012 M520983); Special Projects of China Postdoctoral Science Foundation (2014 T70461); National Student Innovation Training Program of China (1210286090); Natural Science Foundation of Jiangsu Province for Young Scholars (BK2012334); "Summit of the Six Top Talents" Program of Jiangsu Province (2013-WSW-054); Innovative Foundation of Southeast University (3290002401); Wuxi City Science and Technology Development/Medical and Public Health Technology Research and Development Project Funding (CSE31N1517). Jiangsu Provincial Medical Talent, The Project of Invigorating Health Care through Science, Technology, and Education (ZDRCA2016083); The Six Projects Sponsoring Talent Summits of Jiangsu Province, China (LGY2017099).

\section{Availability of data and materials}

All data generated or analyzed during this study are included in this published article [and its supplementary information files].

\section{Authors' contributions}

LS contributed to the experiment design, performed all animal models' TDSC isolation and identification, and wrote the manuscript. YJL contributed to the experiment design, blood glucose measurement, and sample collection and performed the in vitro study. GCD performed the intraperitoneal glucose tolerance test. YCL prepared the paraffin embedding, section, and staining. $\mathrm{CW}$ and $\mathrm{HC}$ contributed to the analysis of the blind staining. GL contributed to the study design and data interpretation. YFR contributed to the study design, data interpretation, and manuscript editing. All authors have read and approved the final submitted manuscript.

\section{Ethics approval}

All animal experiments were approved by the Animal Experimentation Ethics Committee, School of Medicine, Southeast University.

\section{Consent for publication}

Not applicable.

\section{Competing interests}

The authors declare that they have no competing interests.

\section{Publisher's Note}

Springer Nature remains neutral with regard to jurisdictional claims in published maps and institutional affiliations.

\section{Author details}

${ }^{1}$ Department of Orthopaedics, Zhongda Hospital, School of Medicine, Southeast University, No. 87 Ding Jia Qiao, Nanjing 210009, Jiangsu, People's Republic of China. ${ }^{2}$ Orthopaedic Trauma Institute, Southeast University, No. 87 Ding Jia Qiao, Nanjing 210009, Jiangsu, People's Republic of China. ${ }^{3}$ Trauma Center, Zhongda Hospital, School of Medicine, Southeast University, No. 87 Ding Jia Qiao, Nanjing 210009, Jiangsu, People's Republic of China. ${ }^{4}$ Multidisciplinary Team (MDT) for Geriatric Hip Fracture Comprehensive Management, Zhongda Hospital, School of Medicine, Southeast University, No. 87 Ding Jia Qiao, Nanjing 210009, Jiangsu, People's Republic of China. ${ }^{5}$ School of Medicine, Southeast University, No. 87 Ding Jia Qiao, Nanjing 210009, Jiangsu, People's Republic of China. 'Department of Geriatrics, Zhongda Hospital, School of Medicine, Southeast University, 87 Ding Jia Qiao, Nanjing 210009, People's Republic of China. ${ }^{7}$ Department of Orthopaedics and Traumatology, Faculty of Medicine, The Chinese University of Hong Kong, Hong Kong, Hong Kong. ${ }^{8}$ Department of Orthopaedics, Xishan People's Hospital, 588 Guang Rui Road, Wuxi 214011, Jiangsu, People's Republic of China. ${ }^{9}$ China Orthopedic Regenerative Medicine Group, Hangzhou 310000, Zhejiang, People's Republic of China.

Received: 25 September 2018 Revised: 7 December 2018 Accepted: 12 December 2018 Published online: 15 January 2019

References

1. Wong J, Constantino M, Yue DK. Morbidity and mortality in young-onset type 2 diabetes in comparison to type 1 diabetes: where are we now? Curr Diab Rep. 2015:15:566.

2. Stitt AW, Curtis TM. Advanced glycation and retinal pathology during diabetes. Pharmacol Rep. 2005;57(Suppl):156-68.

3. Smit AJ, Gerrits EG. Skin autofluorescence as a measure of advanced glycation endproduct deposition: a novel risk marker in chronic kidney disease. Curr Opin Nephrol Hypertens. 2010;19:527-33.

4. Stolzing A, Sellers D, Llewelyn O, Scutt A. Diabetes induced changes in rat mesenchymal stem cells. Cells Tissues Organs. 2010:191:453-65.

5. Aydeniz A, Gursoy S, Guney E. Which musculoskeletal complications are most frequently seen in type 2 diabetes mellitus? J Int Med Res. 2008;36: 505-11.

6. de Oliveira RR, Lemos A, de Castro Silveira PV, da Silva RJ, de Moraes SR. Alterations of tendons in patients with diabetes mellitus: a systematic review. Diab Med. 2011;28:886-95.

7. Abate M, Salini V, Antinolfi P, Schiavone C. Ultrasound morphology of the Achilles in asymptomatic patients with and without diabetes. Foot Ankle Int. 2014:35:44-9.

8. Akturk M, Ozdemir A, Maral I, Yetkin I, Arslan M. Evaluation of Achilles tendon thickening in type 2 diabetes mellitus. Exp Clin Endocrinol Diab. 2007;115:92-6. 
9. Papanas N, Courcoutsakis N, Papatheodorou K, Daskalogiannakis G, Maltezos E, Prassopoulos P. Achilles tendon volume in type 2 diabetic patients with or without peripheral neuropathy: MRI study. Exp Clin Endocrinol Diab. 2009;117:645-8.

10. Batista F, Nery C, Pinzur M, Monteiro AC, de Souza EF, Felippe FH, Alcantara MC, Campos RS. Achilles tendinopathy in diabetes mellitus. Foot Ankle Int. 2008;29:498-501.

11. Maffulli N, Longo UG, Maffulli GD, Khanna A, Denaro V. Achilles tendon ruptures in diabetic patients. Arch Orthop Trauma Surg. 2011;131:33-8.

12. Fox AJ, Bedi A, Deng XH, Ying L, Harris PE, Warren RF, Rodeo SA. Diabetes mellitus alters the mechanical properties of the native tendon in an experimental rat model. J Orthop Res. 2011;29:880-5.

13. Bedi A, Fox AJ, Harris PE, Deng XH, Ying L, Warren RF, Rodeo SA. Diabetes mellitus impairs tendon-bone healing after rotator cuff repair. J Shoulder Elbow Surg. 2010;19:978-88.

14. de Oliveira RR, Martins CS, Rocha YR, Braga AB, Mattos RM, Hecht F, Brito GA, Nasciutti LE. Experimental diabetes induces structural, inflammatory and vascular changes of Achilles tendons. PLoS One. 2013;8:e74942.

15. Ahmed AS, Schizas N, Li J, Ahmed M, Ostenson CG, Salo P, Hewitt C, Hart DA, Ackermann PW. Type 2 diabetes impairs tendon repair after injury in a rat model. J Appl Physiol. 2012;113:1784-91.

16. Ji J, wang Z, Shi D, Gao X, Jiang Q. Pathologic changes of Achilles tendon in leptin-deficient mice. Rheumatol Int. 2010;30:489-93.

17. Rui YF, Lui PP, Wong YM, Tan Q, Chan KM. Altered fate of tendon-derived stem cells isolated from a failed tendon-healing animal model of tendinopathy. Stem Cells Dev. 2013;22:1076-85.

18. Szkudelski T. The mechanism of alloxan and streptozotocin action in B cells of the rat pancreas. Physiol Res. 2001;50:537-46.

19. Rui YF, Lui PP, Rolf CG, Wong YM, Lee YW, Chan KM. Expression of chondroosteogenic BMPs in clinical samples of patellar tendinopathy. Knee Surg Sports Traumatol Arthrosc. 2012;20:1409-17.

20. Y.F. Rui, P.P. Lui, G. Li, S.C. Fu, Y.W. Lee, K.M. Chan, Isolation and characterization of multipotent rat tendon-derived stem cells, Tissue Eng A, 16 (2010) 1549-1558.

21. Tan Q, Lui PP, Rui YF. Effect of in vitro passaging on the stem cell-related properties of tendon-derived stem cells-implications in tissue engineering. Stem Cells Dev. 2012;21:790-800.

22. Shi L, Rui YF, Li G, Wang C. Alterations of tendons in diabetes mellitus: what are the current findings? Int Orthop. 2015;39:1465-73.

23. Bring DK, Kreicbergs A, Renstrom PA, Ackermann PW. Physical activity modulates nerve plasticity and stimulates repair after Achilles tendon rupture. J Orthop Res. 2007;25:164-72.

24. Kameyama M, Chen KR, Mukai K, Shimada A, Atsumi Y, Yanagimoto S. Histopathological characteristics of stenosing flexor tenosynovitis in diabetic patients and possible associations with diabetes-related variables. J Hand Surg. 2013;38:1331-9.

25. Abate M, Schiavone C, Salini V. Sonographic evaluation of the shoulder in asymptomatic elderly subjects with diabetes. BMC Musculoskelet Disord. 2010;11:278

26. Lin Y-C, Li Y-J, Rui Y-F, Dai G-C, Shi L, Xu H-L, Ni M, Zhao S, Chen H, Wang C, Li G, Teng G-J. The effects of high glucose on tendon-derived stem cells: implications of the pathogenesis of diabetic tendon disorders. Oncotarget. 2017:8:17518-28.

27. Ponta H, Sherman L, Herrlich PA. CD44: from adhesion molecules to signalling regulators. Nat Rev Mol Cell Biol. 2003;4:33-45.

28. Pham PV, Phan NL, Nguyen NT, Truong NH, Duong TT, Le DV, Truong KD, Phan NK. Differentiation of breast cancer stem cells by knockdown of CD44: promising differentiation therapy. J Transl Med. 2011;9:209.

29. Guo J, Chan KM, Zhang JF, Li G. Tendon-derived stem cells undergo spontaneous tenogenic differentiation. Exp Cell Res. 2016;341:1-7.

30. Pryce BA, Brent AE, Murchison ND, Tabin CJ, Schweitzer R. Generation of transgenic tendon reporters, ScxGFP and ScxAP, using regulatory elements of the scleraxis gene. Dev Dyn. 2007;236:1677-82.

31. Humphrey JD, Dufresne ER, Schwartz MA. Mechanotransduction and extracellular matrix homeostasis. Nat Rev Mol Cell Biol. 2014;15:802-12.

32. de Oliveira RR, de Lira KD, Silveira PV, Coutinho MP, Medeiros MN, Teixeira MF, de Moraes SR. Mechanical properties of Achilles tendon in rats induced to experimental diabetes. Ann Biomed Eng. 2011;39:1528-34.

33. Lehner C, Gehwolf R, Wagner A, Resch H, Hirzinger C, Augat P, Stephan D, Aigner L, Rivera FJ, Bauer HC, Tempfer H. Tendons from non-diabetic humans and rats harbor a population of insulin-producing, pancreatic beta cell-like cells. Horm Metab Res. 2012;44:506-10.

34. Schnedl WJ, Ferber S, Johnson JH, Newgard CB. STZ transport and cytotoxicity. Specific enhancement in GLUT2-expressing cells. Diabetes. 1994:43:1326-33.

35. Kishimoto Y, Ohkawara B, Sakai T, Ito M, Masuda A, Ishiguro N, Shukunami C, Docheva D, Ohno K. Wnt/beta-catenin signaling suppresses expressions of Scx, Mkx, and Tnmd in tendon-derived cells. PLoS One. 2017;12:e0182051.

36. Liu X, Chen W, Zhou Y, Tang K, Zhang J. Mechanical tension promotes the osteogenic differentiation of rat tendon-derived stem cells through the Wnt5a/Wnt5b/JNK signaling pathway. Cell Physiol Biochem. 2015;36:517-30.

37. Yuan Z, Li Q, Luo S, Liu Z, Luo D, Zhang B, Zhang D, Rao P, Xiao J. PPARgamma and Wnt signaling in adipogenic and osteogenic differentiation of mesenchymal stem cells. Curr Stem Cell Res Ther. 2016;11: 216-25.

\section{Ready to submit your research? Choose BMC and benefit from:}

- fast, convenient online submission

- thorough peer review by experienced researchers in your field

- rapid publication on acceptance

- support for research data, including large and complex data types

- gold Open Access which fosters wider collaboration and increased citations

- maximum visibility for your research: over $100 \mathrm{M}$ website views per year

At BMC, research is always in progress.

Learn more biomedcentral.com/submissions 